\title{
Enhancement of Global Vehicle Localization using Navigable Road Maps and Dead-Reckoning
}

\author{
Clément Fouque*, Philippe Bonnifait*, David Bétaille ${ }^{\dagger}$ \\ * HeuDiaSyC UMR CNRS 6599, Université de Technologie de Compiègne, France \\ ${ }^{\dagger}$ LCPC, Nantes, France
}

\begin{abstract}
This paper presents a data fusion strategy for the global localization of car-like vehicles. The system uses raw GNSS measurements, dead-reckoning sensors and road map data. We present a new method to use the map information as a heading observation in a Kalman filter. Experimental results show the benefit of such a method when the GPS information is not available. Then, we propose a conservative localization strategy that relies mainly on dead-reckoned navigation. The GNSS measurements and the map information are not used when consistency tests are doubtful. Experimental tests indicate that the performance is effectively better when using only the available consistent information.
\end{abstract}

\section{INTRODUCTION}

Today, providers of Advanced Driver Assistance Systems already offer a wide range of products that most often include itinerary planning and guidance functions, but also real-time rerouting in case of traffic jam. In a near future, the next step forward will consider road safety taking into account road and vehicle physical features, weather conditions, danger awareness and driver behavior. These enhanced applications face, like standard navigation systems, the problem of positioning accuracy, but also that of integrity. Several envisaged applications would expect positioning and mapping at the lane level accuracy, which is still a challenge.

There are many situations in which providing a good vehicle estimated location is impossible using only the GNSS measurements since the receiver can suffer from outages, signal propagation troubles or bad geometrical configuration of satellites. Moreover, most of the recent vehicles are equipped with inertial and odometric sensors accessible through a CAN gateway which allows computing Dead-Reckoning (DR) estimates. Finally, because of the frequent use of navigation systems, navigable maps describing the road network can also be involved in the localization process.

Usually, localization and mapping functions are performed one next the other, with generally no coupling, despite both handle objects (coordinates, pseudo-ranges (PR), road map segments, points of interest...) that could interfere on each other. Some interesting solutions have already been investigated, like using the map of the environment to predict possible masked satellites and outliers in PR due to multipath [1]: this technique needs a priori $3 \mathrm{D}$ modeling on the city, which is still not yet available on a large scale. Another idea has been investigated by [2], [3] that brought about a multi-hypothesis map-matching algorithm where road map segments are used as hard constraint in the localization problem. Approaches in the robotics community are also of great interest. Let us quote the algorithm recently published in [4] that couples MAL (Map Aided Localization) with SLAM (Simultaneous Location And Mapping). Unfortunately, this algorithm supposes landmarks and camera vision, which still makes its application on-board standard vehicles quite difficult.

Our solution will go ahead, since the map is used in the filtering process which improves the capability of the tracking function with the consideration of integrity: the map data is considered as an additional measurement that can be corrupted. In the following, we present a data fusion strategy relying principally on DR under the hypothesis that the DR performances are very good. When the localizer is tracking the pose, the map and the GPS observations are considered as doubtful. They are involved in the localization process when they are consistent with the DR pose. Moreover, when the vehicle approaches an ambiguity zone (detected thanks to the connectivity information of the map), the map information is not taken into account. When leaving the ambiguity zone, the map tracking starts again according to the integrity criterion.

The paper is organized as follows. In the first section, we explain how to formalize the problem by using a local working frame: this is crucial for the tightly coupled approach used to fuse the raw GPS data, the DR sensors and the map data. Then, we present the DR evolution model and we describe the GPS observation models. In section 3, we show how to use the map as a heading constraint since the relative precision of the maps is often good. This is a contribution of this work. We present in section 4 the global fusion strategy relying on integrity tests. Finally, we report, in the last section, real experiments obtained with our lab car using a tightly-coupled Extended Kalman Filter.

\section{SENSOR MODELS}

\section{A. Working frame}

The geographical information stored in navigable road maps (like those provided by NavTeQ and TeleAtlas) is usually expressed in geographic coordinates. We propose to consider only a limited area (called "road cache") around the estimated location. After the road cache extraction, the points of the poly-lines that describe the roads are converted into a local tangent East-North-Up $(E N U)$ frame. Then, by choosing a reference point, the transformation between ECEF (WGS84 
Earth-Centered, Earth-Fixed) and ENU is computed [5]. We denote this transformation by ${ }^{\mathrm{ECEF}} T_{\mathrm{ENU}}$ in homogeneous form. Since the elevation is usually not available in a navigable map, we convert the map points in the working frame by supposing that they are all located at the ellipsoidal height. Regarding the GPS data, only the satellites positions have to be transformed as we use the PR measurements. As the $E N U$ frame is attached to a road cache, it should be noted that the working frame is temporary and valid only for small regions.

\section{B. Evolution model}

As DR measurements are used in the evolution model, the vehicle kinematics $(v, \omega)$ and vehicle heading relative to East $\theta$ are considered in the state vector, in addition to the vehicle pose. The receiver clock bias $d t_{u}$ and the drift $\dot{d} t_{u}$ are also estimated, since raw GNSS data is used in a tightly-coupled strategy. Thus, the estimated state is, in the working frame:

$$
s_{k}=\left[x, y, z, \theta, v, \omega, d t_{u}, \dot{d} t_{u}\right]
$$

Since the origin of the mobile frame is attached to the middle of the rear axle, the motion of the vehicle is supposed to be locally circular in the $(x, y)$ plane of the local frame. The altitude is supposed to remain constant:

$$
\left\{\begin{array}{cccc}
x_{k+1} & =x_{k}+T_{e} v_{k} \cos \left(\theta_{k}+\frac{1}{2} T_{e} w_{k}\right) \\
y_{k+1} & =y_{k}+T_{e} v_{k} \sin \left(\theta_{k}+\frac{1}{2} T_{e} w_{k}\right) \\
z_{k+1} & =z_{k} & \\
\theta_{k+1} & =\theta_{k} & \\
v_{k+1} & =v_{k} & \\
\omega_{k+1} & =w_{k} \\
d t_{k+1} & =d t_{k} & \\
\dot{d} t_{k+1} & =\dot{d} t_{k}
\end{array}\right.
$$

where $T_{e}$ is the sample time. The vehicle motion is depicted by a non-linear equations set, where $\alpha_{k}$ represents the motion model errors:

$$
s_{k+1 \mid k}=f\left(s_{k \mid k}\right)+\alpha_{k}
$$

\section{Measurement models}

In this section, the used sensors and associated measurement models are presented.

1) Dead-Reckoning: The DR sensors provide direct measurements of the vehicle kinematics, by neglecting their response time. Since dead-reckoning is a crucial issue for our method, we suggest that we use a fiber optic yaw rate gyro (a KVH400). With such a sensor it is not necessary to observe the offset. The gyro provides directly the angular velocity of the vehicle. This measurement is denoted $\omega_{m}$.

The rear Wheel Speed Sensors (WSS) of ABS systems provide good estimates of wheel speed under non-slip assumption. With $v_{l}$ (resp. $v_{r}$ ) the left (resp. right) wheel linear speed, the longitudinal velocity $v_{m}$ of the vehicle can be measured by:

$$
v_{m}=\frac{\left(v_{l}+v_{g}\right)}{2}
$$

Therefore, the observation model for the DR is:

$$
\left\{\begin{array}{l}
v_{m}=v \\
\omega_{m}=\omega
\end{array}\right.
$$

2) GPS: The raw GPS measurements are provided by a Septentrio PolarX2e working at $10 \mathrm{~Hz}$. For simplicity purpose, only the PR are used, like when using a low-cost GPS receiver. The PR positioning model for the $i^{t h}$ satellite (denoted SV in the following) is given by:

$$
\rho_{m}^{i}=R^{i}+c\left(d t_{u}-d t_{s}^{i}\right)-\delta_{\mathrm{atm}}^{i}-M^{i}
$$

with:

- $\rho_{m}$ : the measured PR

- $R$ : the geometrical distance between the SV and the receiver

- $d t_{s}$ : the SV clock bias

- $\delta_{\text {atm }}$ : the atmospherical bias

- $M$ : the multipath bias.

The SV clock bias is known from the broadcasted ephemeris, and the atmospherical bias is corrected using either the broadcasted data or WAAS/EGNOS corrections. Moreover, by using this observation model, we assume that the GPS signal is not affected by multipath (normal operation). A trouble of this kind can be detected by the fault detection strategy that we propose to implement. Thus, after applying the corrections, the simplified PR observation model is:

$$
\begin{aligned}
\rho^{i} & =R^{i}+c d t_{u} \\
{\left[\rho^{1}, \ldots, \rho^{n}\right]^{T} } & =h\left(s_{k}\right)
\end{aligned}
$$

The knowledge of the PR variance noise is useful to enhance the navigation loop. When using a WAAS/EGNOS enabled receiver, the broadcasted variance estimate has to be used. Otherwise, a rough noise-measurement model can be computed according to the SV elevation. In a stand alone situation, we propose to use the following formula:

$$
\sigma_{\mathrm{SV}}^{i}=\sigma_{0}\left[1+\tan \left(\frac{\pi}{\pi-2 \psi_{e l}}\left(\frac{\pi}{2}-\psi^{i}\right)\right)\right]
$$

Where $\sigma_{0}$ is the nominal noise variance for a SV at $90^{\circ}$ elevation. $\psi_{e l}$ is the elevation mask angle and $\psi^{i}$ is the elevation angle of $\mathrm{SV}^{i}$. So, a SV near to the mask elevation has huge variance (ie. a small weight in the computation).

\section{NAVIGABle MAP-AIDED OdOMETRY}

\section{A. Navigable Road Maps}

Commonly, a navigable road map is a database that contains a vectorial description of the road network, where each road is described by its discrete center-line. Usually, the data associated to a road is classified into three groups:

- Geometrical: the road shape is described by a poly-line 
- Topological: the connectivity between the roads

- Semantic: road names, speed limit, ...

A map can be used in several ways; a map-matched location of the vehicle can be introduced as a measurement in the localization filter [6] the road can be used for Virtual DGPS [7] some variable can be eliminated by supposing the pose constrained to the segment path [4].

Due to bad absolute precision, map data can suffer from offset with ground truth. Moreover, vehicles rarely evolve exactly on the poly-line describing the road. Therefore, using the map as a position measurement can bias the estimation process that often supposes zero mean errors. It should be noticed that, on the contrary, these biases do not affect the heading information since the map relative precision is often good (always much better than the absolute precision). The main factor affecting the heading information of the map is a low spatial sampling that induces a bad geometry.

Moreover, it is well known that DR drift is very dependent on the quality of the heading estimate. For all these reasons, we propose to use the heading information of the map as an observation is the filtering process.

\section{B. Road Selection}

In order to get a map heading, a first road selection stage has to be addressed. Thanks to the estimated vehicle location, a small area (called a road cache) is extracted from the navigable road database. This has great importance for realtime operation with limited processing resources [8].

So, for each road segment in the cache, a Mahalanobis distance $\Delta_{\text {seg }}^{j}$ is computed between segment $j$ and the estimated pose [9]. It is given by:

$$
\Delta_{\mathrm{seg}}^{j}=\frac{d^{2}}{\sigma_{d}^{2}+\lambda_{\max }^{2}}+\frac{\left(\theta_{s}-\theta_{u}\right)^{2}}{\sigma_{\theta_{s}}^{2}+\sigma_{\theta_{u}}^{2}}
$$

where $d$ is the vehicle-to-segment distance and $\theta_{s}$ the segment heading. $\sigma_{d}$ and $\sigma_{\theta_{s}}$ are the variances associated to these measurements. $\lambda_{\max }$ is the maximum eigen value of the position covariance. Next, the most-likely segment is chosen as the one with the lowest $\Delta_{\text {seg }}^{j}$.

\section{Map Observation}

Considering that the correct road segment has been chosen and that any ambiguous situation is avoided, a map observation can be built. The segment heading is determined using the geometrical points and the driving direction. Since the map data uses the $E N U$ working frame, the segment heading is given relatively to East. By denoting $M\left(m_{1}, m_{2}\right)$ and $N\left(n_{1}, n_{2}\right)$ the extremities of the segment, the map heading angle is given by:

$$
\tan \theta_{s}=\frac{n_{2}-m_{2}}{n_{1}-m_{1}}
$$

The driving direction $\tau$ is then computed according to the segment heading $\theta_{s}$ and the vehicle heading $\theta$ :

$$
\tau=\operatorname{sgn}\left(\frac{\sin \theta_{s}}{\sin \theta}\right)
$$

Therefore, the measured segment heading is given by the following expression:

$$
\left\{\begin{array}{lll}
\theta_{m}=\theta_{s} & \text { if } & \tau=1 \\
\theta_{m}=2 \pi-\theta_{s} & \text { if } & \tau=-1
\end{array}\right.
$$

In addition to this measurement, a standard deviation must be defined for the filtering process. It can be interpreted as a map confidence or weighting factor. A minimal standard deviation $\sigma_{0}$ is set for each segment. Afterwards, $\sigma_{0}$ is modulated by the vehicle speed to indicate in the filter that when the speed is high, the difference between the segment and the vehicle headings should be small. On the contrary, for a small speed the map heading is meaningless, for instance in case of U-turn. Thus, we propose the following formula to modulate the map confidence factor $\sigma_{h}$ :

$$
\left\{\begin{array}{cl}
\sigma_{h}(v)=\left(\frac{\pi}{2}-\sigma_{0}\right) \frac{v}{v_{n}}+\sigma_{0} & \left., \text { if } v \in] 0, v_{n}\right] \\
\sigma_{h}(v)=\sigma_{0} & , \text { otherwise }
\end{array}\right.
$$

with $v_{n}$ a user-defined reference speed.

\section{Navigable Map-Aided Odometry}

This technique consists in applying a Kalman filter that follows the stages:

- Compute a prediction stage with the evolution model described in II-B,

- Do a Kalman correction with the DR measurements,

- Do the road selection and compute the map heading,

- Do a Kalman correction with this observation.

In the experimental section, a test will illustrate the performance of this technique.

\section{GLOBAL FUSION}

The map information is sometimes difficult to handle because of unavoidable ambiguities, at junctions for instance. Moreover, it can suffer sometimes from significant errors when two consecutive mappings are joined end to end. On its side, GNSS measurements can also be degraded by multipath or interferences.

In this section, we present tightly-coupled fusion strategy that proposes to manage these issues. First, we suppose that the DR measurements are always trustworthy. When the estimate is close to a junction, the map is not used. Then, when the localizer is tracking the pose, the map and the GPS observations are considered as doubtful: they are used only when integrity tests are passed. 


\section{A. GNSS Faults Detection}

In real field operation, GNSS signal can suffer from various faults: multipath, atmospheric errors or jamming for instance. Please refer to [10] for more detailed examples. A strategy consists in detecting and isolating the GNSS faults.

A first efficient test can be easily implemented in Kalman filtering by monitoring Normalized Innovation Squared (NIS). This test is performed on all the GNSS measurements using the current estimate $\hat{s}_{k \mid k-1}$. A fault is detected if:

$$
D_{\mathrm{GPS}}>T h_{\mathrm{GPS}}
$$

with:

$$
D_{\mathrm{GPS}}=\left(y-h\left(\hat{s}_{k \mid k-1}\right)\right)^{t} \cdot W \cdot\left(y-h\left(\hat{s}_{k \mid k-1}\right)\right)
$$

where

$$
W=\left(H_{k} \cdot P_{k \mid k-1} \cdot H_{k}^{t}+Q_{b}\right)^{-1}
$$

Assuming that the GNSS signal noise is zero-mean and Gaussian, the decision threshold $T h_{\mathrm{GPS}}$ can be computed according to a $\chi^{2}$ distribution by using a given probability of false alarm $p_{\mathrm{FA}}$ and $n$ degrees of freedom, which is here the number of $\mathrm{SV}$ used. So, $T h_{\mathrm{GPS}}$ is given by:

$$
T h_{\mathrm{GPS}}=\chi^{-2}\left(1-p_{\mathrm{FA}}, n\right)
$$

Moreover, in order to improve our cautious strategy, we propose to reject also the GNSS measurements if the GDOP is greater than 6 .

\section{B. Maps Errors Detection}

As stated before, a navigable road database suffers from geometry errors (road connectivity errors are not considered here). In addition to absolute bias, which does not affect directly the heading information, some road can be missing in the database or the vehicle can be off-road. Moreover, spatial sampling can be insufficient in certain areas due to database simplifications. These simplifications can lead to a poor vehicle heading estimation in a ' $S$ ' curve depicted by only few segments for instance. Hence, the consistency of the selected road segment must be assessed before fusing it in the filter. Using the output of the road selection III-B, the consistency is checked in a same manner as the GNSS data using a Mahalanobis distance corresponding to the selected segment $\left(\Delta_{\text {seg }}\right)$. It is then compared to a decision threshold:

$$
D_{\mathrm{MAP}}>T h_{\mathrm{MAP}}
$$

where:

$$
\left\{\begin{array}{l}
D_{\mathrm{MAP}}=\Delta_{\text {seg }} \\
T h_{\mathrm{MAP}}=\chi^{-2}\left(1-p_{\mathrm{FA}}, 2\right)
\end{array}\right.
$$

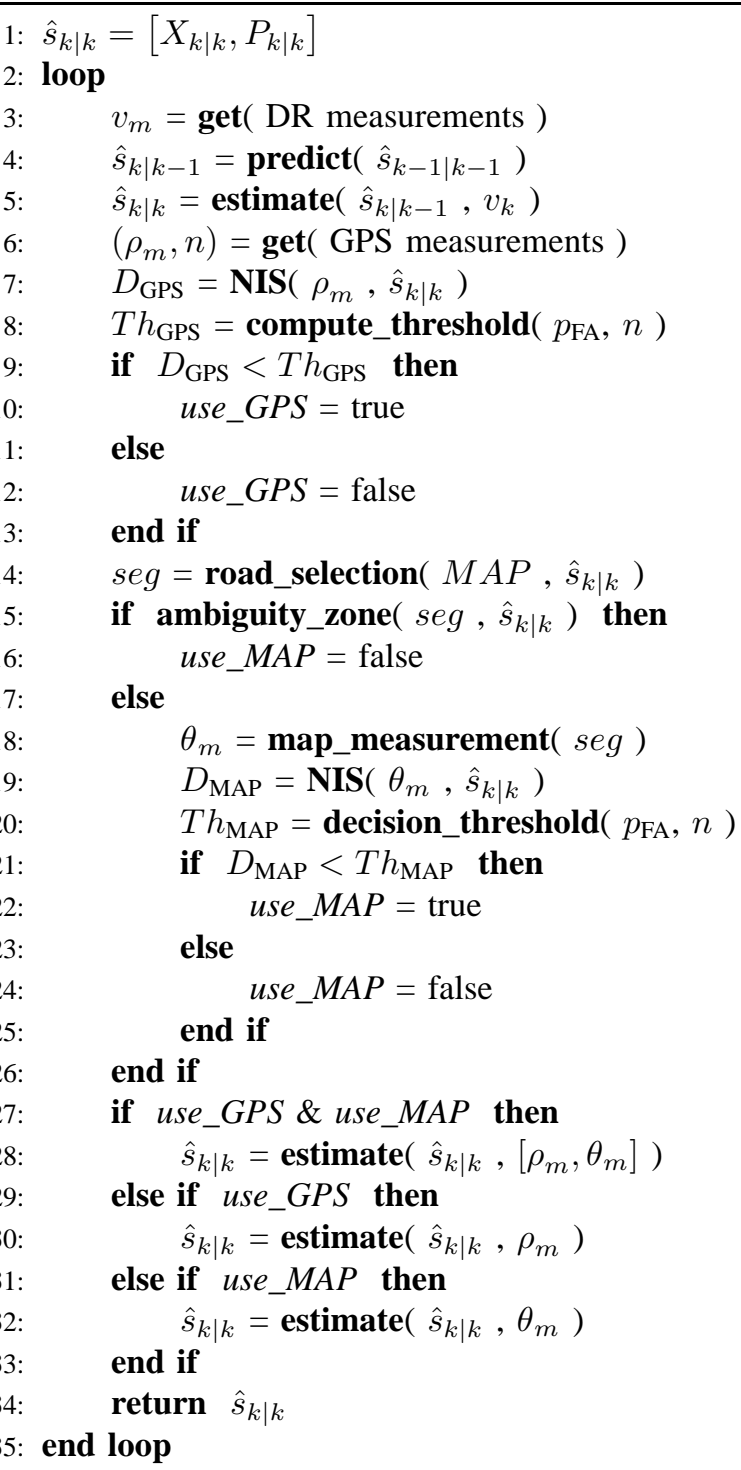

Algorithm 1: Map-aided hybridized GPS algorithm

\section{Kalman-based Algorithm}

The navigation loop relies on an Extended Kalman Filter (EKF) using serialized state estimation stages. At each step, an odometric state is always computed from the DR measurements and the previous estimate state. Using this estimate, the road selection and the test of ambiguity zone are made. If the vehicle is not situated inside an ambiguous area, the consistencies of the map and of the GNSS measurements are examined. Thus, the final estimated $\hat{s}_{k \mid k}$ is updated with the GNSS and/or map measurements according to their availability and integrity.

\section{EXPERIMENTAL RESULTS}

\section{A. Setup and Environment}

Experiments have been carried out in May 2007 using the experimental vehicle Strada (Fig.1). The reference path of the vehicle has been recorded using a DGPS PPK receiver 


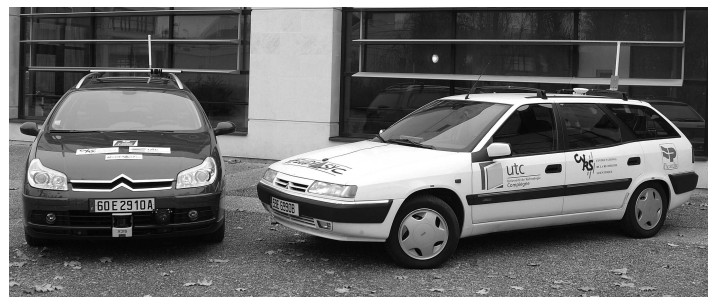

Figure 1. HeuDiaSyC experimental vehicles: Carmen (left) and Strada $($ right $)$

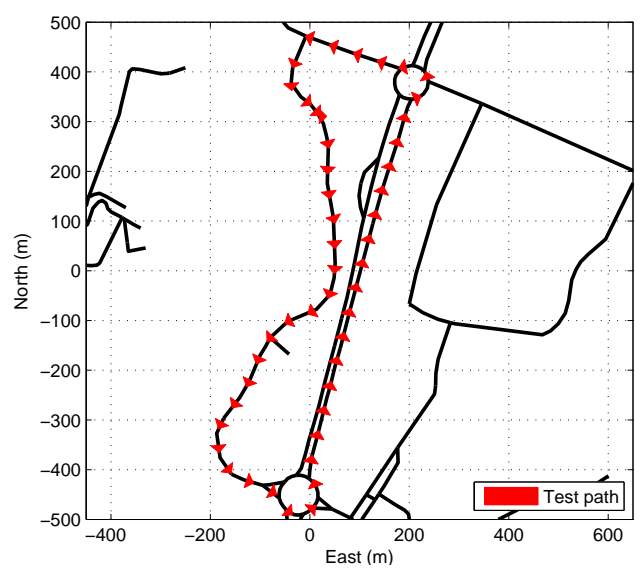

Figure 2. Experimental test track

and 4 bases from the French Orpheon network. The car was driven in Compiegne suburbs, which constitute an open area with occasional tree foliage leading to GPS errors and bad geometrical configuration of the SV constellation.

\section{B. Map-Aided Odometry}

In this first experiment, the benefit of the map heading information on dead-reckoning is illustrated. In this test, the road cache has been reduced to a single road to avoid ambiguous areas (a straight line between two roundabouts, cf. Fig.2). Before tracking the road, the Kalman filter was initialized using the GNSS measurements in an open sky area to avoid GNSS errors. Then, the GNSS information has been forgotten to make the filter navigate using map-aided odometry.

A significant improvement can be seen thanks to the use of the map. Let us consider the $1 \sigma$ variance in the vehicle body-frame depicted in Fig.3. The longitudinal error variance is not presented here as its drift cannot be corrected by the map heading. It can be noticed that the odometric drift can be compensated twice thanks to the use of the map heading. Considering now the map confidence (Fig.3), one can see that the confidence increases as the vehicle accelerates (at the beginning the vehicle is leaving a roundabout). At time index 32 , the map confidence stands at $\sigma_{0}$ because the vehicle speed is higher than $v_{n}\left(v_{n}=20 \mathrm{~m} . \mathrm{s}^{-1}\right)$. After $53 \mathrm{~s}$, the vehicle reaches the end of the road and slows down. Thus, the map confidence decreases according to (14). One can notice that the lateral error drift stands low: it is due to the Kalman Filter
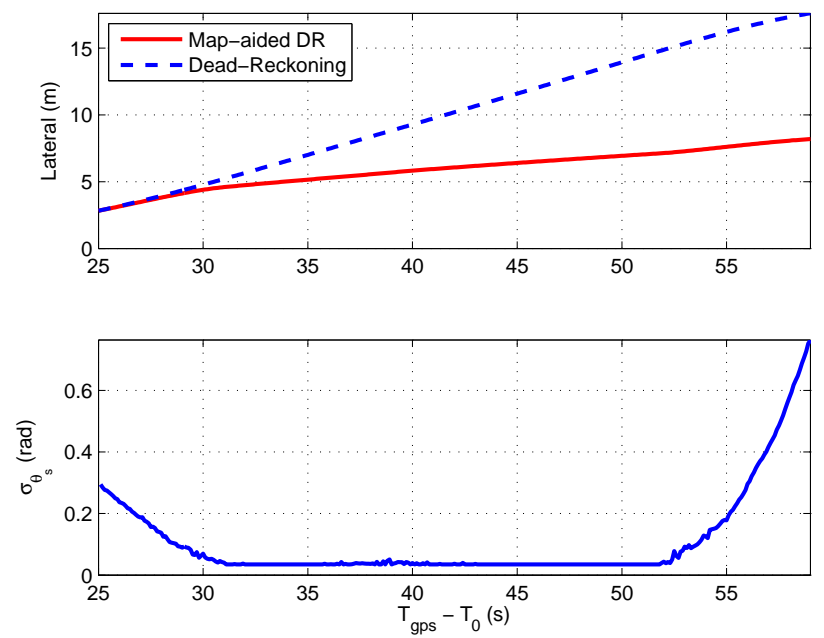

Figure 3. Transversal error variance in the vehicule body-frame (top) and map measurement confidence (down)

dynamics.

\section{Performance of the Complete System}

In this section, the behaviour of the map-hybridized GPS (MGH) is presented. The test was made on a full lap of the presented test track (Fig.2). Along the West section of the map, a huge tree foliage was encountered. Due to this tree foliage, the vehicle location was poorly estimated (Fig.4 and Fig.5). Thus, a large positioning error occurs as the GNSS constellation offers a poor geometry (high GDOP, cf. Fig.7). Moreover, the DGPS PPK was not able to provide a reference position for several time steps while driving this road section.

Fig.7 presents the behaviour of GNSS rejection. One can see here that the GNSS measurements are rejected most of time between the $40^{t h}$ second and the $70^{t h}$ second. This corresponds to the time needed to go across the GNSS disrupted road section. According to Fig.5, a large positioning error can be noted (up to 22 meters). Moreover, this GNSS disturbance also appears on the GNSS measurement innovation (Fig.7) allowing the GNSS signal to be rejected. Therefore, during this period, the map-aided odometry is used to estimate the vehicle location. Hence, the estimated variance of the vehicle position increases but only a small positioning error is made.

After the $70^{t h}$ second, the vehicle enters a wide openarea where no more GNSS faults are encountered according to the low NIS and the good GDOP (Fig.7). According to Fig.5, the MGH error is lower than the GPS error. Providing that the EGNOS broadcasted corrections are not in use, the atmospheric bias is corrected using the data broadcasted by GPS. Thus, these large estimate errors are imputable to a poor estimation of PR biases. According to Fig.6, the estimated variance slowly decreases after entering the open-sky area as the GNSS measurements are in use.

\section{CONCLUSION}

In this paper, the benefits of using a navigable road map for aiding Dead-Reckoning has been presented (Section III). After 


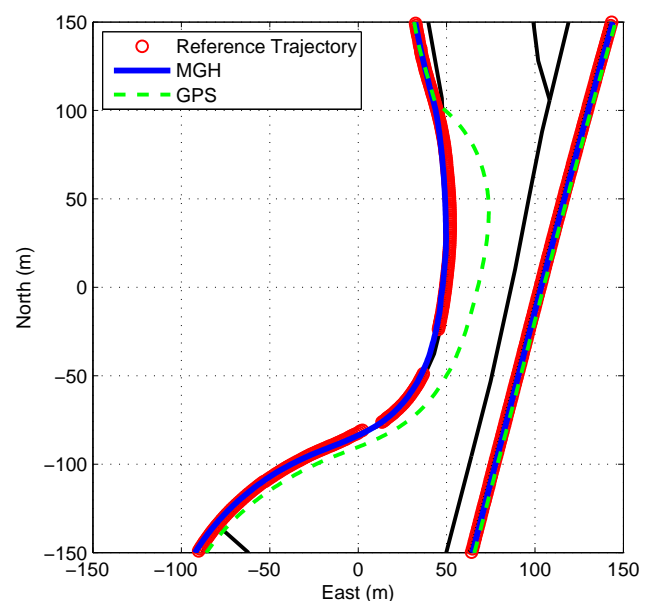

Figure 4. GNSS signal disturbance under tree foliage

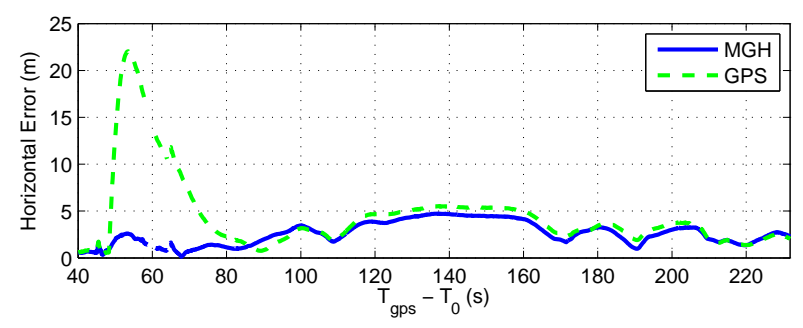

Figure 5. Horizontal quadratic error

position estimation using DR measurements, the map heading is obtained after a road selection. Afterwards, the estimated position is corrected using the map heading. Hence, the lateral odometric drift can be compensated in a significant way thanks to the map heading (Section V-B).

The benefits of a cautious strategy have been also presented. DR measurements are considered as the only reliable measurements. Because of a mono-hypothesis filtering, ambiguous areas are defined according to the road connection. In these particular areas, the map measurement is rejected to avoid miss-matching errors at road junctions. Moreover, the map measurement can be rejected according to the road-selection integrity check. In a same way, GNSS measurements are considered as doubtful. Using the GDOP and a simple Fault Detection strategy, GNSS can be rejected. Therefore, faulty measurements are eliminated before entering the navigation loop, so that its reliability increases.

The future of this research concerns the use of numerical model of terrain in order to provide a height estimate for purpose of reliability improvement. In the same way, the benefits of using SBAS corrections and future GNSS improvement for accuracy and precision of the estimated position will be studied.

\section{REFERENCES}

[1] PoleStar, "Positioning device and method," Patent No.: WO/2006/075116, July 2006.
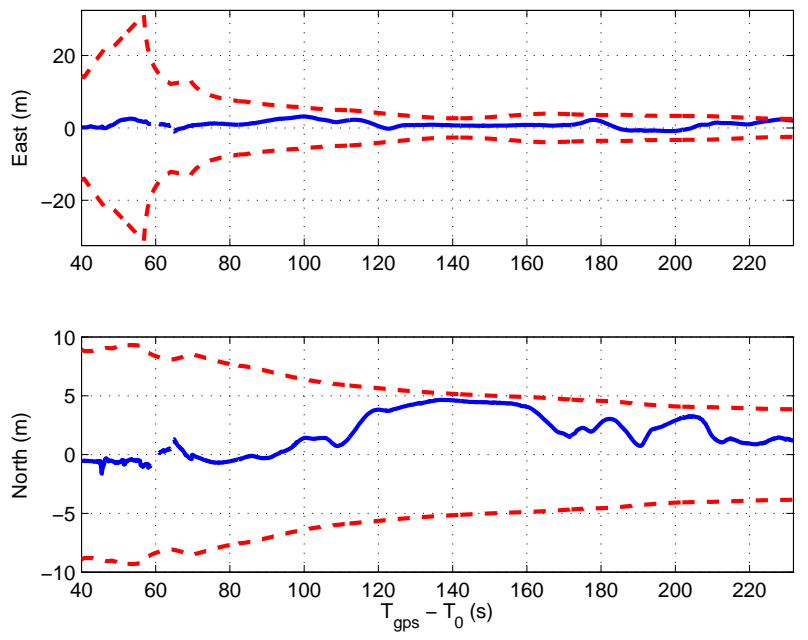

Figure 6. Positioning errors and $3 \sigma$ variance
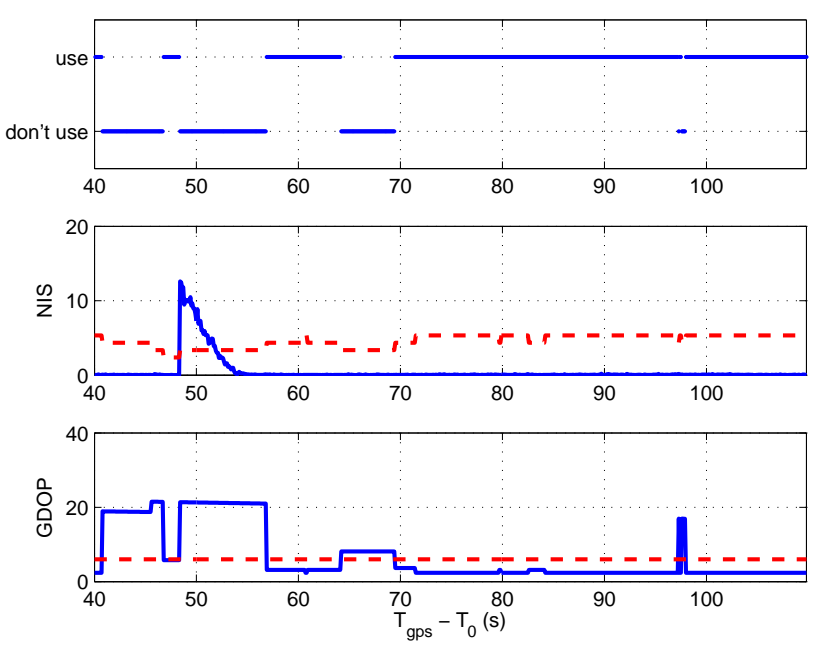

Figure 7. GPS usage

[2] Y. Cui and S. Ge, "Autonomous vehicle positioning with gps in urban canyon environments," IEEE Trans. on Rob. and Aut., vol. 19, pp. 15-25, 2003.

[3] U. Forssell, P. Hall, S. Ahlqvist, and F. Gustafsson, "A novel map-aided positioning system," Proc. of FIS/TA, 2002.

[4] K. W. Lee, S. Wijesoma, and J. I. Guzman, "A constrained slam approach to robust and accurate localisation of autonomous ground vehicles," Robotics and Autonomous Systems, vol. 55, no. 7, pp. 527-540, 2007.

[5] E. D. Kaplan, Understanding GPS: principles and applications, A. House, Ed. Boston, 1996.

[6] H. Sun and M. E. Cannon, "Reliability of an its navigation system," Proceedings of the ITSC Conference, pp. 1040-1046, November 9-11 1997.

[7] G. Taylor and G. Blewitt, "Virtual differential gps and road reduction filtering by map matching," Proceedings of ION'99, pp. 1675-1684, 1999.

[8] P. Bonnifait, M. Jabbour, and G. Dherbomez, "Real-time implementation of a gis-based localization system for intelligent vehicles," EURASIP Journal on Embedded Systems, pp. 12-24, June 2007.

[9] A. Lahrech, C. Boucher, and J.-C. Noyer, "Accurate vehicle positioning in urban areas," IECON Proceedings (Industrial Electronics Conference), vol. 2005, pp. 486-490, 2005.

[10] U. Bhatti and W. Ochieng, "Failure modes and models for integrated gps/ins systems," Journal of Navigation, vol. 60, no. 2, pp. 327-348, 2007. 